\title{
Effect of carglumic acid with or without ammonia scavengers on hyperammonaemia in acute decompensation episodes of organic acidurias
}

Anupam Chakrapani ${ }^{1 *}$, Vassili Valayannopoulos ${ }^{2,9}$, Nuria García Segarra ${ }^{3,10}$, Mireia Del Toro ${ }^{4}$, Maria Alice Donati ${ }^{5}$, Angeles García-Cazorla ${ }^{6}$, María Julieta González ${ }^{6}$, Celine Plisson $^{7}$ and Vincenzo Giordano ${ }^{8}$

\begin{abstract}
Background: Hyperammonaemia is a key sign of decompensation in organic acidurias (OAs) and can contribute to severe neurological complications, thus requiring rapid treatment.

Methods: A post-hoc analysis of two retrospective studies analysed the efficacy of carglumic acid \pm ammonia $\left(\mathrm{NH}_{3}\right)$ scavengers compared with scavengers alone for reducing plasma $\mathrm{NH}_{3}$ levels in patients with $\mathrm{OAs}$ and hyperammonaemia (plasma $\mathrm{NH}_{3}>60 \mu \mathrm{mol} / \mathrm{L}$ ) during decompensation episodes. $\mathrm{NH}_{3}$ was analysed in 12-h periods at 0-48 $\mathrm{h}$ and 24-h periods at 48-120 h. Treatment-emergent adverse events (TEAEs) were recorded.
\end{abstract}

Results: Of 98 episodes, 38 were treated with carglumic acid (34 patients), 33 with $\mathrm{NH}_{3}$ scavengers (22 patients) and 27 with carglumic acid combined with $\mathrm{NH}_{3}$ scavengers (27 patients). Overall, 45\% (carglumic acid group), 46\% $\left(\mathrm{NH}_{3}\right.$ scavengers group) and $74 \%$ (combination group) of episodes occurred in neonates. Median episode duration was 6 days for the carglumic acid and combination groups, and 9 days for the $\mathrm{NH}_{3}$ scavenger group. Median baseline $\mathrm{NH}_{3}$ level was: $199 \mu \mathrm{mol} / \mathrm{L}$, carglumic acid; $122 \mu \mathrm{mol} / \mathrm{L}, \mathrm{NH}_{3}$ scavengers; and $271 \mu \mathrm{mol} / \mathrm{L}$, combination; 13, 30 and $11 \%$ of episodes required extracorporeal detoxification (ED), respectively. Data were censored at ED initiation. While baseline $\mathrm{NH}_{3}$ levels were higher in the combination and carglumic acid groups, mean reduction in $\mathrm{NH}_{3}$ levels to $72 \mathrm{~h}$ in both groups was greater than the $\mathrm{NH}_{3}$ scavengers' group; reductions were greatest in the combination group.

Mean change in plasma $\mathrm{NH}_{3}$ vs baseline in the carglumic acid, $\mathrm{NH}_{3}$ scavengers and combination groups, respectively, was $-13,+12 \%$ and $-27 \%$ at $0-12 \mathrm{~h}\left(p<0.05 \mathrm{NH}_{3}\right.$ scavengers vs combination); $-47,-22 \%$ and $52 \%$ at $12-24 \mathrm{~h}$ (not significant); $-44,-5 \%$ and $-61 \%$ at $24-48 \mathrm{~h}$; and $-66,-16 \%$ and $-76 \%$ at $48-72 \mathrm{~h}(p<0.05$ carglumic acid/combination groups vs $\mathrm{NH}_{3}$ scavengers for both timepoints). The number of TEAEs was similar between groups and mainly related to the disease/condition.

Conclusions: Carglumic acid is a well-tolerated and efficacious treatment for OA decompensation episodes. When given alone or combined with $\mathrm{NH}_{3}$ scavengers, the reduction in $\mathrm{NH}_{3}$ was greater than with $\mathrm{NH}_{3}$ scavengers alone in the first $72 \mathrm{~h}$.

Keywords: Carglumic acid, Ammonia scavengers, Organic aciduria, Hyperammonaemia, Decompensation, Extracorporeal detoxification

\footnotetext{
* Correspondence: anupam.chakrapani@gosh.nhs.uk

${ }^{1}$ Metabolic Medicine Department, Great Ormond Street Hospital NHS

Foundation Trust, Great Ormond Street, London WC1N 3JH, UK

Full list of author information is available at the end of the article
}

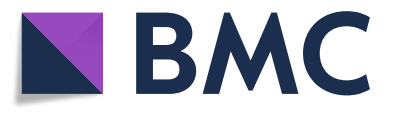

(c) The Author(s). 2018 Open Access This article is distributed under the terms of the Creative Commons Attribution 4.0 International License (http://creativecommons.org/licenses/by/4.0/), which permits unrestricted use, distribution, and reproduction in any medium, provided you give appropriate credit to the original author(s) and the source, provide a link to the Creative Commons license, and indicate if changes were made. The Creative Commons Public Domain Dedication waiver (http://creativecommons.org/publicdomain/zero/1.0/) applies to the data made available in this article, unless otherwise stated. 


\section{Background}

Organic acidurias (OAs) are rare, inherited metabolic disorders, in which impaired metabolism of organic acids results in the build-up of toxic metabolites in the blood, urine and tissues [1, 2]. The classical OAs include three types of inherited disorders of branched-chain amino acids: isovaleric aciduria (IVA), methylmalonic aciduria (MMA) and propionic aciduria (PA) [1] . IVA is caused by mutations in the gene encoding isovaleryl coenzyme A (CoA) dehydrogenase, resulting in defective breakdown of leucine. MMA occurs due to a deficiency of methylmalonyl CoA mutase or due to defects of vitamin B12 metabolism. PA occurs as a result of propionyl CoA carboxylase deficiency. These disorders affect the metabolism of isoleucine, valine, methionine and threonine [1]. Secondary inhibition of the enzyme $\mathrm{N}$-acetylglutamate synthase (NAGS) through accumulation of isovaleryl CoA, methylmalonyl $\mathrm{CoA}$ and propionyl CoA in OAs is thought to be one of the pathogenic mechanisms impeding elimination of ammonia $\left(\mathrm{NH}_{3}\right)$ through the urea cycle, resulting in hyperammonaemia $[2,3]$. In addition, the inability to maintain adequate levels of glutamine precursors secondary to a dysfunctional Krebs' (tricarboxylic acid) cycle due to lack of succinyl CoA synthesis, impaired in both MMA and PA, is also proposed as a mechanism of hyperammonaemia in the OAs [4].

OAs typically manifest in the neonatal period, when they are characterized by toxic encephalopathy presenting within the first few days of life, with symptoms including vomiting, poor feeding and sepsis-like symptoms [5]. If untreated, the condition may progress to lethargy, seizures, coma and multiorgan failure [1]. The most common misdiagnosis of MMA and PA is sepsis. Metabolic acidosis, elevation of lactate and anion gap, urinary ketosis and disturbances of glucose metabolism may help to differentiate MMA and PA from other disorders [6]. The disease course of OA consists of acute metabolic decompensation episodes, during which aspects such as acidosis and hyperammonaemia should be considered. Importantly, these decompensation episodes are medical emergencies and may lead to severe neurological complications if not treated rapidly [1, 7]. A longer duration of hyperammonaemia and higher $\mathrm{NH}_{3}$ levels are associated with poorer neurological outcomes that can lead to serious consequences $[1,5,8,9]$. Therefore, one of the main goals of treatment during OA decompensation episodes is to reduce plasma $\mathrm{NH}_{3}$ levels as quickly as possible $[5,9]$.

Current guidelines recommend various strategies for hyperammonaemia management during OA decompensation episodes, including use of $\mathrm{NH}_{3}$ scavengers and carglumic acid and, in the more severe cases, extracorporeal detoxification (ED) [2]. Ammonia scavengers, such as sodium phenylbutyrate and sodium benzoate, bypass the urea cycle to increase removal of $\mathrm{NH}_{3}$ from the blood, by conjugation of benzoate with glycine to generate hippurate, or phenylacetate with glutamine to generate phenylacetylglutamine $[5,6]$. These conjugates have a higher renal clearance than $\mathrm{NH}_{3}$ itself, and therefore accelerate its excretion in the urine [10].

Carglumic acid is a synthetic structural analogue of $\mathrm{N}$-acetylglutamate (NAG), which promotes $\mathrm{NH}_{3}$ detoxification by mimicking the effects of NAG on carbamoyl-phosphate synthetase I (CPS-I) [2]. CPS-I is a key enzyme of ureagenesis that catalyses the first and rate-limiting step of the urea cycle $[10,11]$. A recent large, retrospective, observational study found that carglumic acid was an efficacious and well-tolerated treatment for hyperammonaemia during OA decompensation episodes [2] . The objective of the current analysis was to further evaluate the specific efficacy of the therapy in reducing raised $\mathrm{NH}_{3}$ levels associated with metabolic decompensation episodes in patients with OAs, without the confounding influences of $\mathrm{NH}_{3}$ scavengers or ED.

\section{Methods}

\section{Study design and patient population}

This was a post-hoc pooled analysis of two retrospective, observational studies. The main results from one of the studies have been published previously [2]. Data were collected from January 1995 to October 2009 in six European countries (Italy, France, Germany, The Netherlands, Spain and the United Kingdom) and Turkey.

Patients were included if they had a confirmed diagnosis of OA and hyperammonaemia (plasma $\mathrm{NH}_{3}>60 \mu \mathrm{mol} / \mathrm{L}$ before treatment), treated for at least one full decompensation episode. Patients with severe hepatic insufficiency at the time of carglumic acid treatment, inherited hepatic malformation or conditions (other than $\mathrm{OA}$ ) that might have contributed to hyperammonaemia were excluded. Mean patient age at baseline in the carglumic acid, $\mathrm{NH}_{3}$ scavenger and combination groups were 34.3 months, 24.6 months, and 19.9 months, respectively.

The study protocols and amendments were approved by the local independent ethics committees (IECs) and/ or institutional review boards. Written informed consent/assent was obtained before data were collected. Cases in which it was not possible to obtain consent (due to death, loss to follow-up) were handled on a case-by-case basis with the relevant IEC. The studies were conducted in accordance with the principles of the Declaration of Helsinki.

\section{Treatments}

Patients were divided into three study groups for analysis based on the treatment that they received: 
carglumic acid (Carbaglu', Orphan Europe, Paris, France) alone; $\mathrm{NH}_{3}$ scavengers (sodium benzoate and/or sodium phenylbutyrate) alone; and carglumic acid combined with $\mathrm{NH}_{3}$ scavengers (combination). Due to the non-interventional, retrospective nature of the studies, oral dosing regimens of carglumic acid were not predefined, and were at the physician's discretion. The recommended initial dose of carglumic acid in Europe (for NAGS deficiency) is $100-250 \mathrm{mg} / \mathrm{kg} /$ day [12]. In this study, the median (Q1, Q3) dose of carglumic acid in the first $24 \mathrm{~h}$ of treatment and the median average daily dose was $101.0 \mathrm{mg} / \mathrm{kg}(62.5,200.0)$ and $97.9 \mathrm{mg} / \mathrm{kg}$ (66.7, 157.9), respectively, in the carglumic acid alone group, and $177.1 \mathrm{mg} / \mathrm{kg}(89.3,256.4)$ and $98.9 \mathrm{mg} / \mathrm{kg}$ (82.6, 164.5), respectively, in the combination group. Treatment with $\mathrm{NH}_{3}$ scavengers was intravenously given in $72 \%$ of episodes, with use of sodium benzoate $(66.7 \%$ of episodes), sodium phenylbutyrate (7.4\% of episodes), or their combination (25.9\% of episodes). The median dose of sodium benzoate was $257.8(149-790) \mathrm{mg} / \mathrm{kg}$ and the median (range) dose of sodium phenylbutyrate was $282.0(169-5625) \mathrm{mg} / \mathrm{kg}$. In the combination group, the median doses of the $\mathrm{NH}_{3}$ scavengers were similar to the median doses in the $\mathrm{NH}_{3}$ group.

Medications of interest initiated to treat the episode were recorded. The most common treatment in all treatment groups was carnitine (90 out of 98 episodes, $91.8 \%$ ). Other common treatments included arginine $(11.2 \%$ of episodes), cobalamin (33.7\% of episodes), glucose $(25.5 \%$ of episodes), biotin ( $28.6 \%$ of episodes), thiamine (8.2\% of episodes), and riboflavin (4.1\% episodes).

\section{Outcomes}

Data on decompensation episode characteristics, plasma $\mathrm{NH}_{3}$ levels and clinical symptoms were collected. The primary outcome was the reduction in plasma $\mathrm{NH}_{3}$ level from 0 to $120 \mathrm{~h}$. Other outcomes included time to success (time to first of two consecutive measurements of plasma $\mathrm{NH}_{3} \leq 60 \mu \mathrm{mol} / \mathrm{L}$ without initiation of ED, death or study withdrawal), time to $50 \%$ reduction in $\mathrm{NH}_{3}$ from baseline (most recent measurement prior to treatment initiation) and the shift in clinical symptoms from baseline to endpoint (last available measurement $\leq 18 \mathrm{~h}$ after the last treatment intake, or Day 15, whichever was earlier). Treatment-emergent adverse events (TEAEs) were recorded.

\section{Statistical analysis}

Statistical analyses were performed, with continuous variables being summarized by descriptive statistics and categorical data presented by absolute and relative frequencies. Efficacy evaluations were conducted on the full analysis set, which included all decompensation episodes from patients who received at least one dose of study treatment and had a confirmed diagnosis of IVA, MMA or PA. Safety analyses were undertaken on the safety set, which included all decompensation episodes from patients who received at least one dose of study treatment.

Plasma $\mathrm{NH}_{3}$ was analysed in 12-h periods from 0 to $48 \mathrm{~h}$ and in 24-h periods from 48 to $120 \mathrm{~h}$. The maximum $\mathrm{NH}_{3}$ value was selected for each time period. The evaluation window was $\leq 15$ days from the first administration of treatment. $\mathrm{NH}_{3}$ data were censored at ED (haemodialysis/haemofiltration/peritoneal dialysis) initiation.

Statistical analyses were conducted using the Statistical Analysis Systems $\left(\mathrm{SAS}^{\circ}\right)$ software versions 9.2/9.3 (SAS Institute, Cary, Northern Carolina, USA) and Adclin ${ }^{\circ}$ software version TPF 3.2.2 (Adclin S.A., Paris, France).

\section{Results}

\section{Episodes and treatments}

In total, 98 episodes in 83 patients were included in the full analysis set: 38 episodes were treated with carglumic acid ( $n=34$ patients), 33 with $\mathrm{NH}_{3}$ scavengers $(n=22$ patients) and 27 with a combination of both ( $n=27$ patients). The episode disposition is shown in Fig. 1. The baseline episode characteristics are displayed in Table 1. The majority of episodes (61.2\%) in all treatment groups occurred in patients with a diagnosis of PA, and the proportion of episodes was higher in the $\mathrm{NH}_{3}$ scavengers' group. More than $80 \%$ of episodes lasted fewer than 15 days, with a median episode duration of 6.0 days in the carglumic acid alone and combination groups, and 8.5 days in the $\mathrm{NH}_{3}$ scavengers' group. Median age at start of the episode was lower, and baseline $\mathrm{NH}_{3}$ level was higher in the combination group than in the carglumic acid alone group or the $\mathrm{NH}_{3}$ scavengers' group (Table 1). The majority (81.6\%) of episodes did not require ED.

The median duration of treatment was 4.0 days for both the carglumic acid alone and the $\mathrm{NH}_{3}$ scavengers' groups, and 5.0 days for the combination group. Sodium benzoate was the most commonly used concomitant $\mathrm{NH}_{3}$ scavenger (alone in $66.7 \%$ of episodes and in combination with sodium phenylbutyrate in $25.9 \%$ of episodes); the median (range) total dose was $257.8 \mathrm{mg} / \mathrm{kg}$ (149-790). Sodium phenylbutyrate was used alone in $7.4 \%$ of episodes; the median (range) total dose was $282.0 \mathrm{mg} / \mathrm{kg}(168.6-562.5)$.

\section{Change in plasma $\mathrm{NH}_{3}$}

Daily plasma $\mathrm{NH}_{3}$ levels are presented in Fig. 2 and the reductions in levels from baseline during the first $72 \mathrm{~h}$ of treatment are displayed in Fig. 3. Up to $72 \mathrm{~h}$, the mean reduction in $\mathrm{NH}_{3}$ from baseline in the carglumic acid alone and combination groups was greater than in the $\mathrm{NH}_{3}$ scavengers' group, where both these groups had 


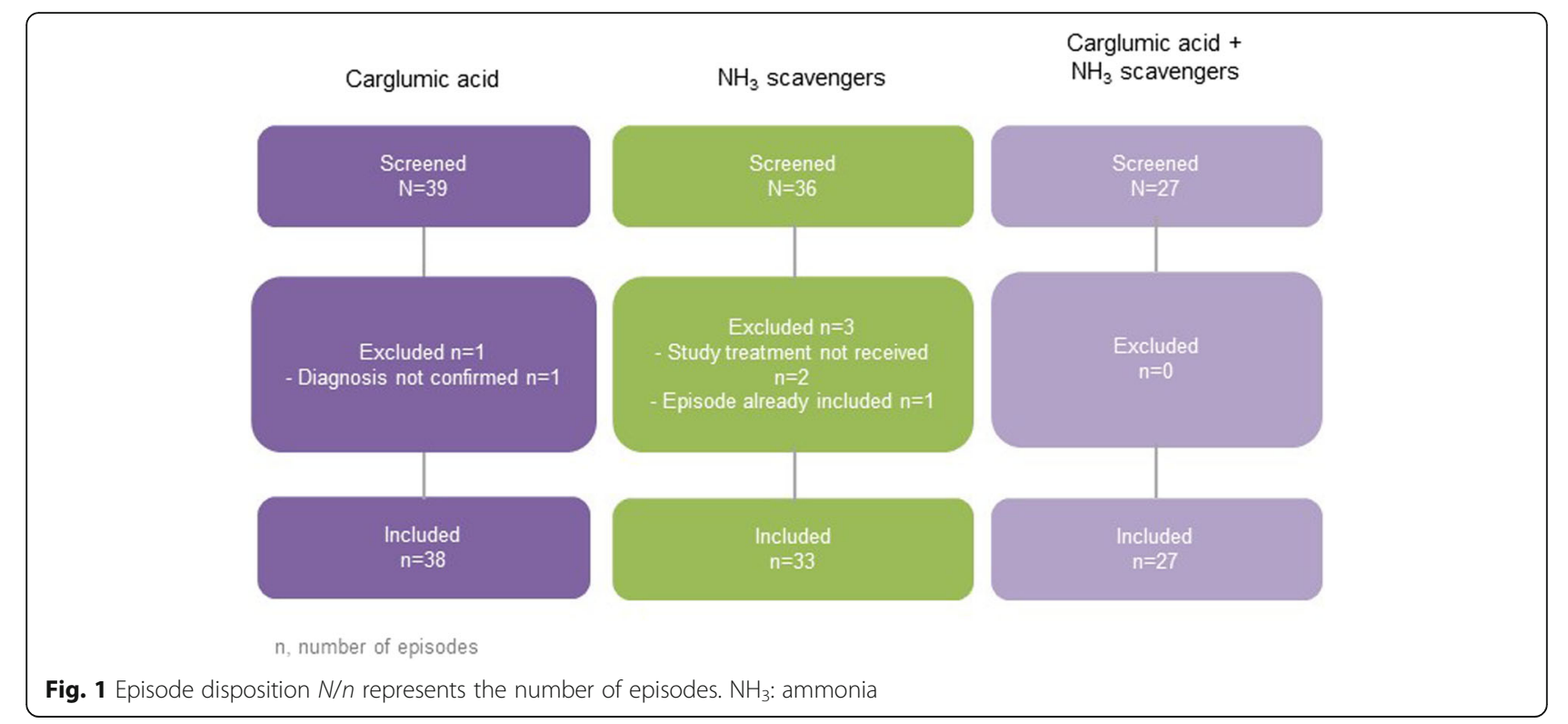

higher baseline $\mathrm{NH}_{3}$ levels. The reductions were greatest in the combination group, in which significantly greater reductions in plasma $\mathrm{NH}_{3}$ were observed versus $\mathrm{NH}_{3}$ scavengers alone at all timepoints, except 12-24 h. Reductions in baseline plasma $\mathrm{NH}_{3}$ were similar for both age categories in the carglumic acid group, and a greater reduction in plasma $\mathrm{NH}_{3}$ was observed for neonates in the combination group when compared with non-neonates. The reductions in plasma $\mathrm{NH}_{3}$ over $72 \mathrm{~h}$ of treatment with carglumic acid alone or in combination with $\mathrm{NH}_{3}$ scavengers were similar for patients with IVA, MMA and PA.
Time to success in $25 \%$ of episodes was $34 \mathrm{~h}$ for the carglumic acid group, $37 \mathrm{~h}$ for the $\mathrm{NH}_{3}$ scavengers' group and $29 \mathrm{~h}$ for the combination group. The median time to reduce baseline $\mathrm{NH}_{3}$ levels by $50 \%$ is shown in Fig. 4. In the combination group, the time to halve baseline $\mathrm{NH}_{3}$ levels was shorter when the average dose of carglumic acid was $\geq 100 \mathrm{mg} / \mathrm{kg}$ than when the average dose was $<100 \mathrm{mg} / \mathrm{kg}$ in the first $24 \mathrm{~h}$.

\section{Change in clinical symptoms}

A summary of the neurological findings and feeding difficulties observed in $>10 \%$ of episodes is presented in

Table 1 Episode characteristics at baseline

\begin{tabular}{|c|c|c|c|}
\hline & Carglumic acid & $\mathrm{NH}_{3}$ scavengers & Carglumic acid $+\mathrm{NH}_{3}$ scavengers \\
\hline Episodes, $n$ & 38 & 33 & 27 \\
\hline Episodes occurring in males, $n(\%)$ & $22(57.9)$ & $16(48.5)$ & $12(44.4)$ \\
\hline Episodes occurring in neonates, $n(\%)$ & $17(44.7)$ & $15(45.5)$ & $20(74.1)$ \\
\hline \multicolumn{4}{|l|}{ Episodes with OA diagnosis, $n$ (\%) } \\
\hline PA & $19(50.0)$ & $27(81.8)$ & $14(51.9)$ \\
\hline MMA & $15(39.5)$ & $5(15.2)$ & $12(44.4)$ \\
\hline IVA & $4(10.5)$ & $1(3.0)$ & $1(3.7)$ \\
\hline Median (Q1, Q3) age at diagnosis, days & $\begin{array}{l}13.0 \\
(5.0,33.0)\end{array}$ & $\begin{array}{l}9.5 \\
(5.0,19.5)\end{array}$ & $\begin{array}{l}8.0 \\
(5.0,15.0)\end{array}$ \\
\hline Median (Q1, Q3) $\mathrm{NH}_{3}$ levels, $\mu \mathrm{mol} / \mathrm{L}$ & $\begin{array}{l}199.0 \\
(125.0,295.0)\end{array}$ & $\begin{array}{l}122.0 \\
(91.0,191.0)\end{array}$ & $\begin{array}{l}270.9 \\
(160.0,429.0)\end{array}$ \\
\hline Median (Q1, Q3) age at start of episode, days & $\begin{array}{l}50.5 \\
(4.0,1190.0)\end{array}$ & $\begin{array}{l}68.0 \\
(4.0,1477.0)\end{array}$ & $\begin{array}{l}5.0 \\
(4.0,61.0)\end{array}$ \\
\hline Median (Q1, Q3) duration of ended episode, days & $\begin{array}{l}6.0 \\
(3.5,8.0)\end{array}$ & $\begin{array}{l}8.5 \\
(5.0,12.0)\end{array}$ & $\begin{array}{l}6.0 \\
(4.0,7.0)\end{array}$ \\
\hline Episodes requiring extracorporeal detoxification, ${ }^{a} n(\%)$ & $5(13.2)$ & $10(30.3)$ & $3(11.1)$ \\
\hline
\end{tabular}

IVA Isovaleric aciduria, MMA Methylmalonic aciduria, $\mathrm{NH}_{3}$ Ammonia, $\mathrm{OA}$ Organic aciduria, PA Propionic aciduria ${ }^{a}$ Haemodialysis, haemofiltration and peritoneal dialysis. Full analysis set 


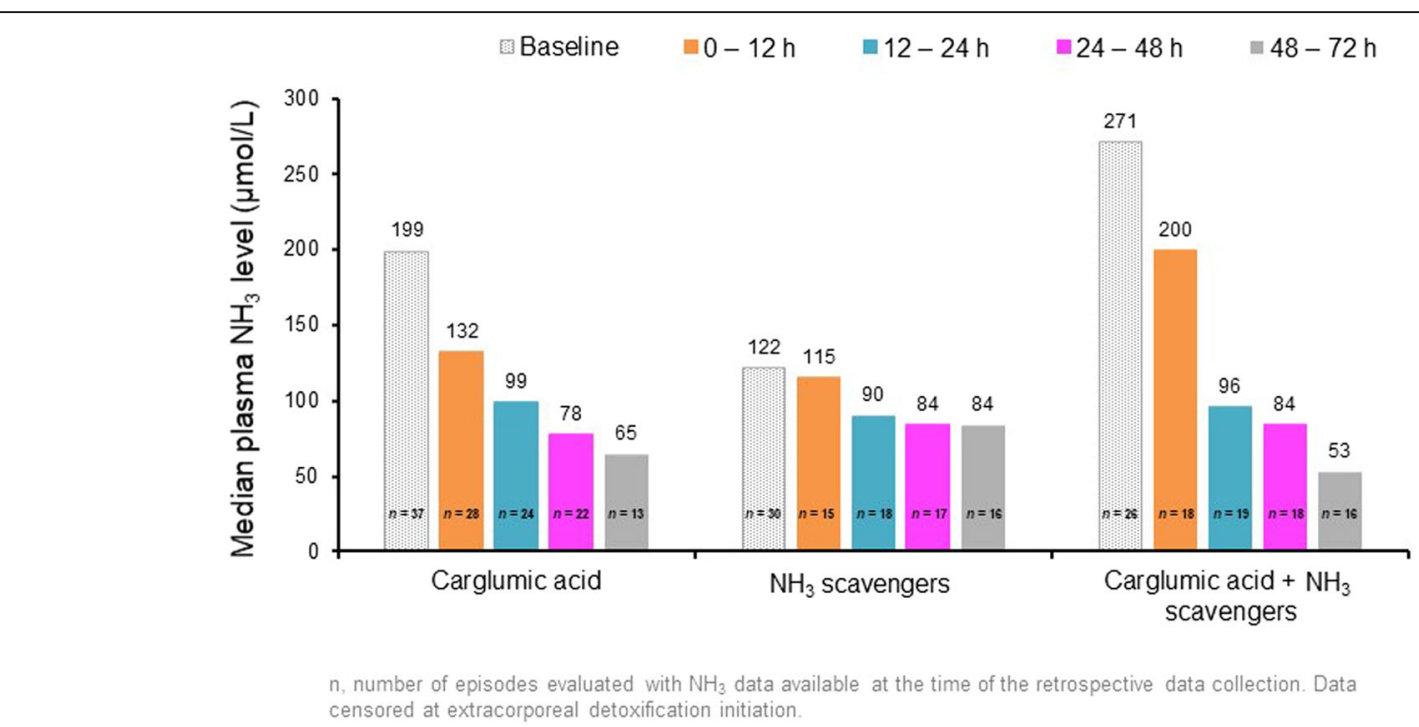

Fig. 2 Median plasma $\mathrm{NH}_{3}$ levels during the first $72 \mathrm{~h}$ of treatment. Data were censored at extracorporeal detoxification initiation. $n$ represents the number of episodes evaluated at the time of the retrospective data collection. h: hour; $\mathrm{NH}_{3}$ : ammonia

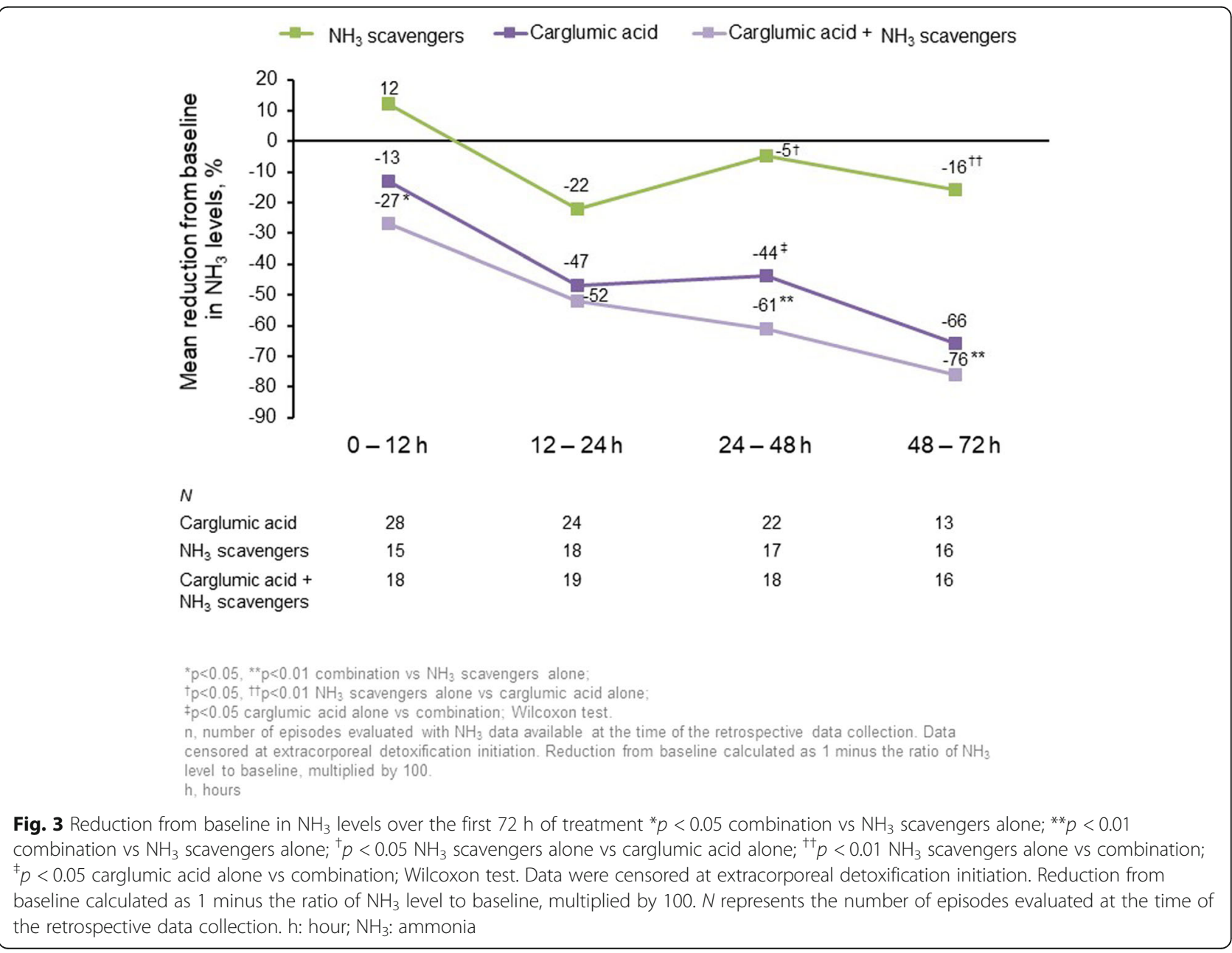




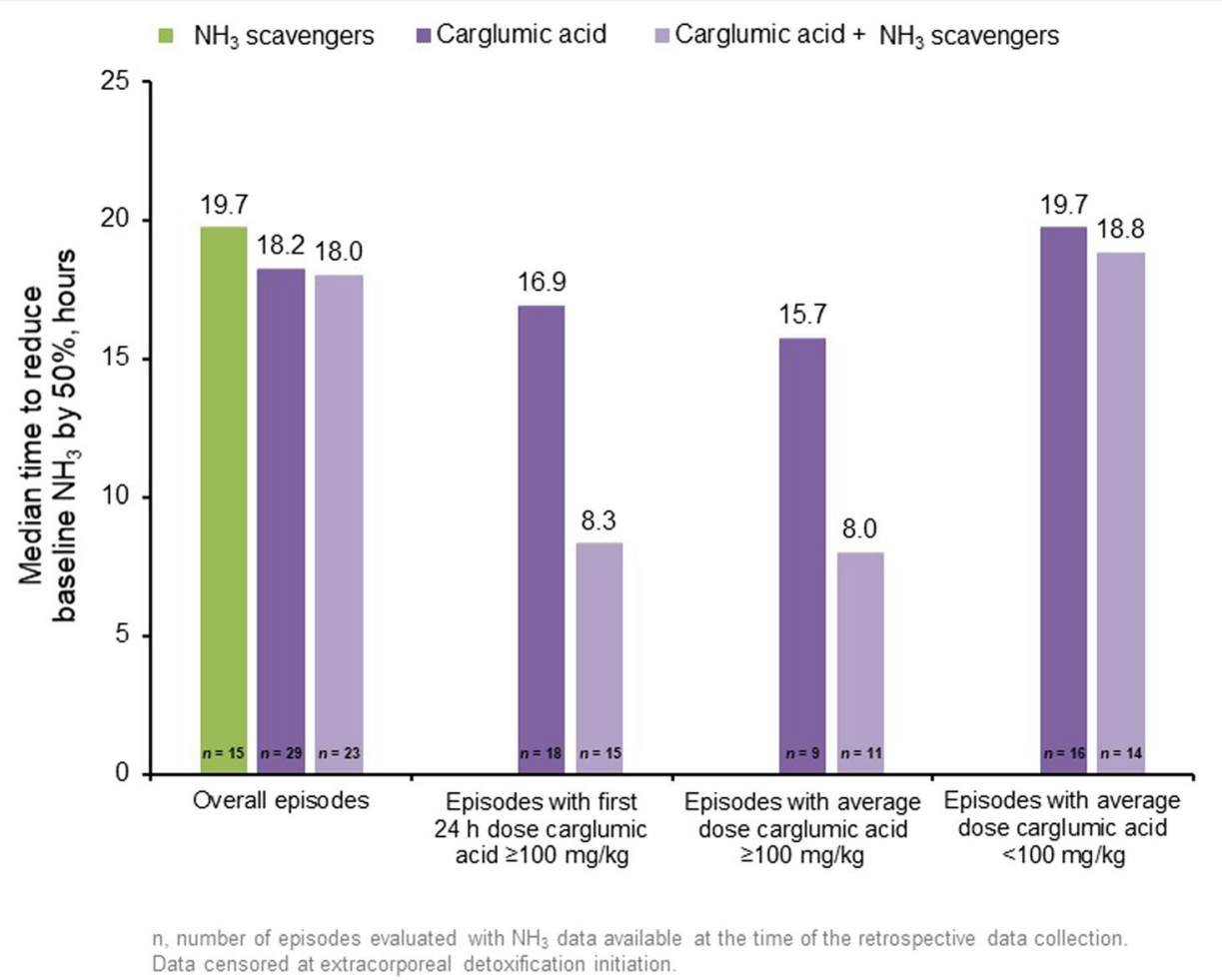

Fig. 4 Time to reduce baseline $\mathrm{NH}_{3}$ levels by $50 \%$ according to the carglumic acid dose. Data were censored at extracorporeal detoxification initiation. Numbers represent the number of episodes evaluated at the time of the retrospective data collection. h: hour; $\mathrm{NH}_{3}$ : ammonia

Table 2. For the majority of symptom categories, there was a marked reduction in symptoms from baseline to study endpoint. At baseline, almost all episodes were associated with neurological findings: carglumic acid alone, $86 \% ; \mathrm{NH}_{3}$ scavengers alone, $92 \%$; combination, $100 \%$. By study endpoint, normal neurological status was reported for $50 \%(9 / 18)$ of episodes in the carglumic acid group, $36 \%(4 / 11)$ of episodes in the $\mathrm{NH}_{3}$ scavengers' group and $45 \%(5 / 11)$ of episodes in the combination group. One patient in the carglumic acid alone group and one patient in the $\mathrm{NH}_{3}$ scavengers' group had no neurological symptoms at baseline but developed symptoms by the study endpoint.

The initial manifestation of episodes was most commonly feeding difficulties, with $90 \%$ of cases in the carglumic acid group, $86 \%$ in the $\mathrm{NH}_{3}$ scavengers' group and $100 \%$ of cases in the combination group presenting with such symptoms. As was seen with neurological findings, a notable shift towards normal feeding was observed with treatment, in 58\% (11/19), $75 \%(9 / 12)$ and $80 \%(8 / 10)$ of episodes in the carglumic acid, $\mathrm{NH}_{3}$ scavengers and combination groups, respectively.

\section{Safety}

Overall, there were fewer adverse events (AEs) in the combination group $(n=21)$ than in the carglumic acid group $(n=61)$ or the $\mathrm{NH}_{3}$ scavengers' group $(n=97)$.
One-hundred and fifty seven TEAEs were reported, including 51 serious TEAEs (Table 3 ) -54 and 13 in the carglumic acid group, 85 and 31 in the $\mathrm{NH}_{3}$ scavengers' group, and 18 and 7 in the combination group, respectively. Overall, the AEs reported were largely related to the disease/condition (i.e. metabolic decompensation) rather than drug toxicity. A total of 22 individual fatal TEAEs that could each have resulted in death occurred in 10 patients/episodes. Three fatal AEs occurred in three patients in the carglumic acid group; 13 fatal AEs occurred in four patients in the $\mathrm{NH}_{3}$ scavengers' group; and six fatal AEs occurred in three patients in the combination group.

\section{Discussion}

Hyperammonaemia is one of the most severe, life-threatening symptoms in OA metabolic decompensation episodes [6]. Acute hyperammonaemia is a medical emergency and early, rapid reduction of plasma $\mathrm{NH}_{3}$ levels, using both pharmacological treatments and non-pharmacological methods available, is required to limit the potential unfavourable neurological symptoms encountered in patients with $\mathrm{OA}$, as well as to reduce the risk of fatal outcomes $[5,6,13]$. Treatment with $\mathrm{NH}_{3}$ scavengers may be beneficial by increasing the reduction of plasma $\mathrm{NH}_{3}$ levels; however, caution is advised when using in MMA and PA due to potential $\mathrm{NH}_{3}$ 
Table 2 Summary of neurological findings and feeding difficulties observed in > 10\% of episodes in any treatment group at baseline

\begin{tabular}{|c|c|c|c|c|c|c|c|}
\hline & \multirow[t]{2}{*}{ Symptoms } & \multicolumn{2}{|c|}{$\begin{array}{l}\text { Carglumic acid episodes } \\
(n=38)\end{array}$} & \multicolumn{2}{|c|}{$\begin{array}{l}\mathrm{NH}_{3} \text { scavengers episodes } \\
(n=33)\end{array}$} & \multicolumn{2}{|c|}{$\begin{array}{l}\text { Carglumic acid }+\mathrm{NH}_{3} \\
\text { scavengers episodes } \\
(n=27)\end{array}$} \\
\hline & & $\begin{array}{l}\text { Baseline } \\
n \text { episodes } \\
(\%)\end{array}$ & $\begin{array}{l}\text { Endpoint } \\
n \text { episodes } \\
(\%)\end{array}$ & $\begin{array}{l}\text { Baseline } \\
n \text { episodes } \\
(\%)\end{array}$ & $\begin{array}{l}\text { Endpoint } \\
n \text { episodes } \\
(\%)\end{array}$ & $\begin{array}{l}\text { Baseline } \\
n \text { episodes } \\
(\%)\end{array}$ & $\begin{array}{l}\text { Endpoint } \\
n \text { episodes } \\
(\%)\end{array}$ \\
\hline \multirow{8}{*}{$\begin{array}{l}\text { Neurological } \\
\text { findings }\end{array}$} & Abnormal movements & $10(26.3)$ & $1(2.6)$ & $3(9.1)$ & $1(3.0)$ & $5(18.5)$ & $1(3.7)$ \\
\hline & Coma & $6(15.8)$ & $1(2.6)$ & $1(3.0)$ & 0 & $3(11.1)$ & $2(7.4)$ \\
\hline & Lethargy & $16(42.1)$ & $4(10.5)$ & $11(33.3)$ & $2(6.1)$ & $17(63.0)$ & $2(7.4)$ \\
\hline & Muscle hypotonia & $15(39.5)$ & $7(18.4)$ & $12(36.4)$ & $1(3.0)$ & $19(70.4)$ & $4(14.8)$ \\
\hline & $\begin{array}{l}\text { Neurological development } \\
\text { impairment }\end{array}$ & $2(5.3)$ & $1(2.6)$ & $4(12.1)$ & $3(9.1)$ & 0 & 0 \\
\hline & Seizures & $2(5.3)$ & 0 & $4(12.1)$ & $1(3.0)$ & $3(11.1)$ & 0 \\
\hline & Somnolence/ asthenia & $18(47.4)$ & $3(7.9)$ & $8(24.2)$ & $1(3.0)$ & $21(77.8)$ & $2(7.4)$ \\
\hline & Visual impairment & $4(10.5)$ & $3(7.9)$ & $1(3.0)$ & $1(3.0)$ & $1(3.7)$ & $2(7.4)^{a}$ \\
\hline \multirow{2}{*}{$\begin{array}{l}\text { Feeding } \\
\text { difficulties }\end{array}$} & Poor feeding & $15(39.5)$ & $4(10.5)$ & $13(39.4)$ & 0 & $18(66.7)$ & $1(3.7)$ \\
\hline & Vomiting & $19(50.0)$ & $7(18.4)$ & 19 (57.6) & $3(9.1)$ & $10(37.0)$ & $1(3.7)$ \\
\hline
\end{tabular}

Full analysis set. $N(\%)$ based on the number of episodes

${ }^{a}$ For one patient, neurological status at baseline was erroneously marked as normal despite the patient having an ongoing history of blindness recorded at screening. The patient's neurological status at endpoint correctly identified visual impairment. $\mathrm{NH}_{3}$ data was censored at extracorporeal detoxification initiation $\mathrm{NH}_{3}$ ammonia

toxicity by blocking the urea cycle through sequestration of CoA [6, 14, 15]. Additionally, sodium phenylbutyrate acts through conjugation of glutamine, and may worsen the glutamine depletion that is one of the proposed mechanisms of hyperammonaemia and energy depletion in the OAs [6]. Therefore, sodium phenylbutyrate is not preferred in the treatment of hyperammonaemia in the OAs $[6,16]$. Furthermore, treatment with these agents at high doses may increase serum sodium and decrease serum potassium levels $[15,17]$. In particularly severe hyperammonaemia, or when treatment with other methods has failed, ED by haemodialysis, haemofiltration or peritoneal dialysis may also be required $[5,6]$. Caution is recommended when using $\mathrm{ED}$, as the procedure is invasive, has a risk of technical failure, and can cause infectious and haemodynamic complications in infants, and use of ED is limited by local facilities $[5,6]$.

The efficacy and safety of carglumic acid during decompensation episodes in OAs has been explored in our previous publication [2]. This analysis aimed to evaluate the efficacy of carglumic acid and its tolerability when used with or without $\mathrm{NH}_{3}$ scavengers, for the treatment

Table 3 Treatment-emergent adverse events

\begin{tabular}{|c|c|c|c|}
\hline & Carglumic acid $(n=38)$ & $\mathrm{NH}_{3}$ scavengers $(n=33)$ & Carglumic acid $+\mathrm{NH}_{3}$ scavengers $(n=27)$ \\
\hline Number of TEAEs & 54 & 85 & 18 \\
\hline Number of drug-related ${ }^{a}$ TEAEs & 18 & 1 & 6 \\
\hline Number of serious TEAEs & 13 & 31 & 7 \\
\hline Number of drug-related ${ }^{\mathrm{b}}$ serious TEAEs & 5 & 0 & 1 \\
\hline TEAEs leading to death & 3 & 13 & 6 \\
\hline \multicolumn{4}{|c|}{ TEAEs occurring in $\geq 10 \%$ of episodes in any treatment group, $n$ episodes (\%) } \\
\hline Coagulopathy & $1(2.6)^{b}$ & $5(15.2)$ & 0 \\
\hline Thrombocytopenia & $2(5.3)^{b}$ & $7(21.2)$ & $1(3.7)$ \\
\hline Hyperglycaemia & $1(2.6)$ & $5(15.2)$ & $1(3.7)$ \\
\hline Hypocalcaemia & $1(2.6)^{b}$ & $4(12.1)$ & 0 \\
\hline Hypokalaemia & $1(2.6)$ & $6(18.2)$ & 0 \\
\hline Respiratory distress & 0 & $4(12.1)$ & 0 \\
\hline
\end{tabular}

$\mathrm{NH}_{3}$ Ammonia, TEAE Treatment-emergent adverse event

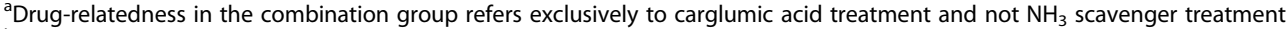

${ }^{\mathrm{b}}$ Considered to be drug-related in one episode/patient. $n$ represents the number of episodes evaluated 
of hyperammonaemia during OA decompensation episodes. The data in this analysis demonstrate that carglumic acid is efficacious in reducing $\mathrm{NH}_{3}$ levels, and produces greater reductions in plasma $\mathrm{NH}_{3}$ levels than $\mathrm{NH}_{3}$ scavengers alone during the first $72 \mathrm{~h}$ of metabolic decompensation episodes. Reductions in plasma $\mathrm{NH}_{3}$ following treatment were accompanied by improvements in clinical symptoms and neurological status. More significant reductions in $\mathrm{NH}_{3}$ were observed in the combination group. This could be attributed to the higher dosage of carglumic acid administered within the first $24 \mathrm{~h}$ or may suggest a potentially greater clinical impact of combination therapy compared with either $\mathrm{NH}_{3}$ scavengers or carglumic acid alone. Reductions in ammonia levels may appear to be more pronounced in the groups where patients had higher baseline levels. It is worth noting that differences in local practice may also reflect the higher percentage of episodes treated via ED within the $\mathrm{NH}_{3}$ scavengers' group compared with the carglumic acid or combination groups.

The higher baseline plasma $\mathrm{NH}_{3}$ levels reported for the combination group were also closer to the range associated with irreversible neurological deficits following prolonged exposure and, therefore, these reductions might be more clinically relevant [8].

Conversely, the baseline plasma $\mathrm{NH}_{3}$ levels in the carglumic acid and $\mathrm{NH}_{3}$ scavengers' group were less elevated, and data from this study do not demonstrate if the relative reduction reported will translate into a clinically meaningful improvement in a patient with a higher baseline plasma $\mathrm{NH}_{3}$ level. Analysis of these findings for patients with IVA was also limited by the low number of IVA patients included in the study; further investigation is required for the use of carglumic acid and $\mathrm{NH}_{3}$ scavengers in this population.

This exploratory analysis suggests that the reduction of $\mathrm{NH}_{3}$ levels may be more rapid with carglumic acid treatment than with $\mathrm{NH}_{3}$ scavengers; this is particularly important during the first hours of an episode in order to limit potential complications. The time to reduce baseline $\mathrm{NH}_{3}$ by $50 \%$ was shortest in patients who received a first 24-h dose or average daily dose of carglumic acid $\geq 100 \mathrm{mg} / \mathrm{kg}$, which is in line with the recommended dose for the treatment (100-250 mg/kg) [18]. More episodes required treatment with ED in the $\mathrm{NH}_{3}$ scavengers alone group than in the carglumic acid alone or combination groups $(30.3,13.2$ and $11.1 \%$, respectively). It is possible that the more rapid reductions in plasma $\mathrm{NH}_{3}$ observed in the carglumic acid groups may have led to a decision not to initiate ED.

Overall, treatment with carglumic acid was well tolerated. There were fewer TEAEs in the combination group than in the carglumic acid and $\mathrm{NH}_{3}$ scavengers monotherapy groups, and the reported TEAEs were mostly related to the condition rather than drug toxicity. The categories of drug relatedness were different between the two studies analysed, and relatedness in the combination group referred exclusively to carglumic acid and not $\mathrm{NH}_{3}$ scavengers. These results suggest that, in addition to improved efficacy in the reduction of plasma $\mathrm{NH}_{3}$ levels, use of a combination of carglumic acid and $\mathrm{NH}_{3}$ scavengers may also reduce the risk of AEs in some patients.

This was a retrospective, exploratory analysis, with missing data for a number of patients for some timepoints, and without control groups. We acknowledge these shortcomings and that they would best be addressed in a study with a controlled and prospective design.

However, the study provides real-world data on the use of carglumic acid with or without $\mathrm{NH}_{3}$ scavengers for the treatment of metabolic decompensation episodes. Data from this analysis are consistent with previous published data supporting the use of carglumic acid in acute hyperammonaemia to reduce plasma $\mathrm{NH}_{3}$ and the need for peritoneal dialysis and haemodialysis [10].

\section{Conclusions}

The results of this analysis indicate that carglumic acid, either alone or combined with $\mathrm{NH}_{3}$ scavengers, produces greater reductions in plasma $\mathrm{NH}_{3}$ levels than $\mathrm{NH}_{3}$ scavengers alone in episodes of hyperammonaemia occurring in metabolic decompensation in patients with OAs. These analyses support the early use of carglumic acid in the treatment of acute hyperammonaemia in paediatric patients with OA.

\section{Abbreviations}

AE: Adverse event; CoA: Coenzyme A; CPS-I: Carbamoyl-phosphate synthetase I; ED: Extracorporeal detoxification; IEC: Independent ethics committee; IVA: Isovaleric aciduria; MMA: MethyImalonic aciduria; NAG: N-acetylglutamate; NAGS: N-acetylglutamate synthase; $\mathrm{NH}_{3}$ : Ammonia; OA: Organic aciduria; PA: Propionic aciduria; TEAE: Treatment-emergent adverse event

\section{Acknowledgements}

Medical writing support was provided by ApotheCom Ltd., London, UK, funded by Orphan Europe.

\section{Funding}

These studies were funded by Orphan Europe. Orphan Europe was involved in the study design, analysis and interpretation of the data, and manuscript preparation.

\section{Availability of data and materials}

The datasets used and/or analysed during the current study are available from the corresponding author on reasonable request.

\section{Authors' contributions}

AC served as Principal Investigator, analysed and interpreted the data, was involved in drafting the manuscript and reviewing the final version. W served as a Principal Investigator for this study, analysed and interpreted the data and contributed in drafting the manuscript. NGS provided the data from the patients from the References Center for Metabolic Diseases at Robert Debré Hospital in Paris. MDT provided data from the patients from the Reference Center for Metabolic Diseases at Hospital d'Hebron in Barcelona. MAD provided the data from the patients from the Reference Center for Inherited Metabolic and Muscolar Diseases at Azienda Ospedale Universitaria Meyer, Florence. AGC provided the data from the patients from 
the Reference Center for Metabolic Diseases at Hospital Sant Joan de Déu in Barcelona. MJG worked with AGC to provide the data from the patients from the Reference Center for Metabolic Diseases at Hospital Sant Joan de Déu in Barcelona, and revised the clinical reports of many patients. CP: was involved in the drafting of the article as well as in the graph preparation and critical analysis. VG has been involved in drafting and revising the manuscript critically for analysis and interpretation of the data. All authors read and reviewed the final manuscript before submission.

\section{Ethics approval and consent to participate}

The study protocols and amendments were approved by the local independent ethics committees (IECS) and/or institutional review boards. Written informed consent/assent was obtained before data were collected. Cases in which it was not possible to obtain consent (due to death, loss to follow-up) were handled on a case-by-case basis with the relevant IEC. The studies were conducted in accordance with the principles of the Declaration of Helsinki.

\section{Consent for publication}

Not applicable.

\section{Competing interests}

$W$ received Principal Investigator fees from Orphan Europe for conducting this study while at Necker-Enfants Malades Hospital. W is currently an employee of Sanofi Genzyme Corporation, Cambridge, MA. CP, JLM and VG are employees of Orphan Europe.

\section{Publisher's Note}

Springer Nature remains neutral with regard to jurisdictional claims in published maps and institutional affiliations.

\section{Author details}

'Metabolic Medicine Department, Great Ormond Street Hospital NHS Foundation Trust, Great Ormond Street, London WC1N 3JH, UK. ${ }^{2}$ Hôpital Necker-Enfants Malades, Paris, France. ${ }^{3}$ Reference Centre for Inherited Metabolic Diseases, Hôpital Robert Debré, Paris, France. ${ }^{4}$ Servicio de Neurologíia Infantil, Hospital Vall d'Hebrón, Barcelona, Spain. ${ }^{5}$ Reference Centre for Inherited Metabolic and Muscular Disease, Azienda Ospedaliero Universitaria Meyer, Florence, Italy. ${ }^{6}$ Hospital Sant Joan de Déu and CIBERER, Instituto de Salud Carlos III, Barcelona, Spain. ${ }^{7}$ Medical Affairs, Orphan Europe, Paris, France. ${ }^{8}$ Orphan Europe, Paris, France. ${ }^{9}$ Present Address: Sanofi Genzyme Corporation, Cambridge, MA, USA. ${ }^{10}$ Present Address: Centre Pédiatrique de Meyrin, Meyrin, Switzerland.

Received: 13 July 2017 Accepted: 11 June 2018

Published online: 20 June 2018

\section{References}

1. Dionisi-Vici C, et al. 'Classical' organic acidurias, propionic aciduria, methylmalonic aciduria and isovaleric aciduria: long-term outcome and effects of expanded newborn screening using tandem mass spectrometry. J Inherit Metab Dis. 2006;29(2-3):383-9.

2. Valayannopoulos $V$, et al. Carglumic acid enhances rapid ammonia detoxification in classical organic acidurias with a favourable risk-benefit profile: a retrospective observational study. Orphanet J Rare Dis. 2016;11:32.

3. Dercksen $\mathrm{M}$, et al. Inhibition of $\mathrm{N}$-acetylglutamate synthase by various monocarboxylic and dicarboxylic short-chain coenzyme a esters and the production of alternative glutamate esters. Biochim Biophys Acta. 2014; 1842(12 Pt A):2510-6.

4. Filipowicz HR, et al. Metabolic changes associated with hyperammonemia in patients with propionic acidemia. Mol Genet Metab. 2006;88(2):123-30.

5. Haberle J, et al. Suggested guidelines for the diagnosis and management of urea cycle disorders. Orphanet J Rare Dis. 2012;7:32.

6. Baumgartner MR, et al. Proposed guidelines for the diagnosis and management of methylmalonic and propionic acidemia. Orphanet J Rare Dis. 2014;9:130.

7. Filippi $\mathrm{L}$, et al. $\mathrm{N}$-carbamylglutamate in emergency management of hyperammonemia in neonatal acute onset propionic and methylmalonic aciduria. Neonatology. 2010;97(3):286-90.

8. Bachmann C. Long-term outcome of patients with urea cycle disorders and the question of neonatal screening. Eur J Pediatr. 2003;162(Suppl 1):S29-33.
9. Walker $\mathrm{V}$. Ammonia toxicity and its prevention in inherited defects of the urea cycle. Diabetes Obes Metab. 2009;11(9):823-35.

10. Daniotti $M$, et al. New developments in the treatment of hyperammonemia: emerging use of carglumic acid. Int J Gen Med. 2011:4:21-8.

11. Ah Mew N, et al. N-carbamylglutamate augments ureagenesis and reduces ammonia and glutamine in propionic acidemia. Pediatrics. 2010;126(1): e208-14.

12. Europe, O., Carbaglu summary of product characteristics. 2017.

13. Haberle J. Clinical and biochemical aspects of primary and secondary hyperammonemic disorders. Arch Biochem Biophys. 2013;536(2):101-8.

14. Cyr D, Maswoswe S, Tremblay G. Inhibition of the urea cycle and de novo pyrimidine biosynthesis by sodium benzoate. J Inherit Metab Dis. 1987; 10(Suppl 2):308-10.

15. Zschocke J, Hoffman G. Diagnosis and management of metabolic disorders, in Vademecum metabolicum diagnosis and treatment of inborn errors of metabolism. Germany: Schattauer Gmbh; 2011.

16. European registry and network for Intoxication type Metabolic Diseases (EIMD), Urea cycle disorders: Quick reference guide, in http://www.e-imd.org/ en/healthcare-professionals/european-proposed-guidelines-using-signmethodology/index.phtml. Accessed 8 May 2017. 2014.

17. Summar M. Current strategies for the management of neonatal urea cycle disorders. J Pediatr. 2001:138(1 Suppl):S30-9.

18. Orphan Europe. Carbaglu summary of product characteristics. 2016.

\section{Ready to submit your research? Choose BMC and benefit from:}

- fast, convenient online submission

- thorough peer review by experienced researchers in your field

- rapid publication on acceptance

- support for research data, including large and complex data types

- gold Open Access which fosters wider collaboration and increased citations

- maximum visibility for your research: over $100 \mathrm{M}$ website views per year

At BMC, research is always in progress.

Learn more biomedcentral.com/submissions 